\title{
Modelling of Urban Traffic Flow
}

\author{
Sharif E. Guseynov ${ }^{2,3,4, b}$, Alexander V. Berezhnoy ${ }^{1,2, a}$ \\ ${ }^{1}$ Faculty of Information Technologies, Ventspils University College, Inženieru Street 101a, Ventspils LV-3601, \\ Latvia; "Entelgine" Research \& Advisory Co., Ltd., Kleistu Street 2-53, Riga LV-1067, Latvia; ${ }^{3}$ Institute of \\ Fundamental Science and Innovative Technologies, Liepaja University, Liepaja LV-3401, Latvia; ${ }^{4}$ Faculty of \\ Science and Engineering, Liepaja University, 4 Kr.Valdemar Street, Liepaja LV-3401, Latvia; \\ aalexander.v.berezhnoy@gmail.com, sh.e.guseinov@inbox.lv
}

\begin{abstract}
In this paper non-deterministic motion of urban traffic is studied under certain assumptions. Based on those assumptions discrete and continuous mathematical models are developed: continuous model is written as the Cauchy initial-value problem for the integro-differential equation, whence among other things it is obtained the Fokker-Planck equation. Besides, the sufficient condition ensuring the mathematical legitimacy of the developed continuous model is formulated.
\end{abstract}

Keywords: traffic flow, mathematical model, Cauchy initial-value problem.

\section{INTRODUCTION}

The main classical question of the traffic flows theory, which is of essential interest also to the contemporary applied sphere of urban traffic management, is to study the existing relationship between the basic physical characteristics of the traffic flow - density, flux and velocity ([1]). The question of exact and unambiguous determination of the main physical characteristics of a traffic flow, in particular, of traffic flow density, is considered to be the most significant question. For this purpose, as a rule, various mathematical models (for instance, see [1]-[9] and respective references given in these), describing the behavior of traffic flow under various conditions are used. The unequivocal finding of the traffic flow density in a desired point of given area at any point in time will allow to exercise more effective and resource-saving management of traffic flows ([10]). In addition, the unambiguous finding of the traffic flow density in the studied areas of the urban transport system will allow changing the controlled parameters of the transport system in such way, so that the vehicles distribution both in the scale of individual road sections, in the scale of certain residential districts as well as in the whole city scale would become admissible, i.e. traffic flows density in the "bottlenecks" road sections during the rush hours were staying within admissible limits, the excess of which leads to various negative consequences, for example, such as formation of traffic jams. One of the greatest difficulties encountered in mathematical modeling of traffic flow, is that the behavior of vehicles on the one hand, must be subject to the restrictions and requirements of the traffic regulations, and on the other hand, is determined by aspiration to achieve the individual goals of the drivers in the general traffic flow. Therefore, in the model there should be introduced additional assumptions, which are allowing not to consider an individual contribution of each possible factor that may affect the dynamics of changes in road conditions, but do not result in distortion of the resulting system behavior as a whole. For this reason, the concept of an equilibrium condition of transport system ([10]) is introduced while constructing mathematical model of the non-deterministic motion of traffic flow. Traffic tends to return to the equilibrium state in the event of possible local deviations. The equilibrium state of the transport system requires that the average flow rate (traffic velocity) at each time point corresponds to its equilibrium value at a given density of vehicles. The specified requirement stated as assumption considerably narrows a scope of application of mathematical models, being limited to consideration of traffic flow only at road sections without crossings, etc. The fundamental work [1] describes in details the main properties of traffic flow (uncertainty, finiteness, dependence of the distance on time and so forth), which ignoring, does not allow to consider the constructed mathematical models as rigorous and real enough that they could be taken as full-fledged traffic models.

This paper considers a non-deterministic motion of urban traffic flow on the assumption that the vehicle can move both forward and backward. At every fixed period of time there are no limitations imposed on vehicles motion within traffic flow, i.e. there are no restrictions imposed that a change of the current location of any vehicle can be carried out only at the adjacent position: such a restriction, as shown in [10], means the traffic flow with preference, and it 
leads to linear mathematical models (both discrete and continuous). In this paper, we give up the abovementioned restriction, which means the following: at every fixed period of time vehicles in traffic flow can replace any of the current locations to any other with a given probability, where the motion to free neighboring location represents just one of the possible particular cases. Such non-deterministic traffic flow will be called traffic "without preference". From a mathematical point of view, the assumption of traffic flow "without preference" is equivalent to the following four assumptions:

- at each moment of time $t=0, \Delta t, 2 \cdot \Delta t, 3 \cdot \Delta t, \ldots$ any of the vehicles may have one of the arbitrary coordinates $0, \pm \Delta x, \pm 2 \cdot \Delta x, \pm 3 \cdot \Delta x, \ldots$;

- if some vehicle at the time moment $n \cdot \Delta t\left(n \in \mathbb{Z}_{+}\right)$has a coordinate $i \cdot \Delta x(i \in \mathbb{Z})$, then at the subsequent time moment $(n+1) \cdot \Delta t$ the same vehicle can be at any (but not only at adjacent with $i \cdot \Delta x$ coordinate $(i-1) \cdot \Delta x$ or neighboring $(i+1) \cdot \Delta x)$ of these coordinates with probability $p_{n ; i, j} \stackrel{\text { def }}{\equiv} p(n ; i, j)$;

- for each vehicle in the traffic flow the probability $p_{n ; i, j}$ that the vehicle, which had at the time moment $n \cdot \Delta t\left(n \in \mathbb{Z}_{+}\right)$coordinate $i \cdot \Delta x(i \in \mathbb{Z})$, would be at the coordinate $j \cdot \Delta x(j \in \mathbb{Z})$ at the next time moment $(n+1) \cdot \Delta t\left(n \in \mathbb{Z}_{+}\right)$, has a Markov character, i.e., $p_{n ; i, j}$ is subject to Markov process: which means that for each vehicle in a traffic flow these probabilities $p_{n ; i, j}\left(n \in \mathbb{Z}_{+} ; i, j \in \mathbb{Z}\right)$ do not depend neither on the state of transport system in the previous time moments, nor on behavior of other vehicles in the same traffic flow;

- the studied traffic flow is assumed uniform (or nearly uniform), and physical properties of the traffic flow - density, intensity and velocity are supposed to be not dependent on the direction of vehicles motion in the transport system.

As it will be shown in the following sections, the above listed four assumptions allow to construct a discrete model, and then, using the principles of continuum mechanics, proceed to the continuous mathematical model for the unknown traffic density.

\section{MODELLING OF THE NON-DETERMINISTIC MOTION OF TRAFFIC FLOW "WITHOUT PREFERENCE"}

The first of the four assumptions outlined in the introduction of this work, along with another assumption (see [10]) that if any vehicle in time moment $n \cdot \Delta t$ has a coordinate $i \cdot \Delta x$, in the subsequent moment of time $(n+1) \cdot \Delta t$ the same vehicle may have a coordinate $(i-1) \cdot \Delta x$ or $(i+1) \cdot \Delta x$, wherein the probability of finding vehicle in these two points are equal, i.e. $P\{x=(i-1) \cdot \Delta x\}=P\{x=(i+1) \cdot \Delta x\}=\frac{1}{2}, \quad$ that leads to the discrete linear model

$$
U(m, n+1) \approx \frac{U(m-1, n)+U(m+1, n)}{2},
$$

where function $U(m, n)\left(n \in \mathbb{Z}_{+} ; m \in \mathbb{Z}\right)$ means the number of vehicles at time moment $n \cdot \Delta t$ at the point having coordinate $m \cdot \Delta x$. For a given value of the initial distribution of vehicles $U(m, 0)$ in the traffic flow, the discrete linear model (1) makes it possible to find approximately the required distribution of vehicles in traffic at all subsequent moments of time. Furthermore, from (1) follows the property that specifies the nature of vehicles distribution changes with time: when the number of vehicles at any point becomes smaller than the arithmetic mean values at neighboring points, then this number increases, and vice versa (in [10] this property has been called the traffic flow restoration property). Thus, the first of the four assumptions mentioned in the introduction of this work generates a linearity property: by adding the initial distributions of vehicles in traffic flow, their distributions at any subsequent time are added as well. Looking ahead, let us note that the latter assumption (i.e., the fourth assumption) will not be active during the construction of the mathematical model, however, this assumption would be essential during the study of already constructed mathematical model, namely, during the proof that constructed mathematical model carries catholicity property in some sense (see the next section). The second and third assumptions, as it will be shown below, radically change the basis of the traffic flow probabilistic process ([10]), which supports the construction of a mathematical model (1). Indeed, first of all, let us note that in view of the fact that in the traffic flow vehicle has to appear somewhere, it could be written

$$
\sum_{j \in \mathbb{Z}} p_{n ; i, j} \equiv 1 \forall\left(n \in \mathbb{Z}_{+}, i \in \mathbb{Z}\right) .
$$

However, by analogy with the identity (15) it could not be stated that $\sum_{i \in \mathbb{Z}} p_{n ; i, j}=1$, because the value $\sum_{i \in \mathbb{Z}} p_{n ; i, j}$ may have a value greater than one, if the 
point $j \cdot \Delta x(j \in \mathbb{Z})$ of the considered section of a one-dimensional road $[-l,+l]$ having a length $2 \cdot l$ is preferable for the driver; and this sum can be less than one in case when the point $j \cdot \Delta x(j \in \mathbb{Z})$ is something not preferable for the driver. Within the time interval $[n \cdot \Delta t, n \cdot \Delta t+\Delta t]\left(n \in \mathbb{Z}_{+}\right)$the number of vehicles moving from the point $m_{1} \cdot \Delta x\left(m_{1} \in \mathbb{Z}\right)$ of the studied one-dimensional road segment having $2 \cdot l$ length to any other point $m_{2} \cdot \Delta x$ $\left(m_{2} \in \mathbb{Z} ; m_{2} \neq m_{1}\right)$ in this section, is equal to $U\left(m_{1}, n\right) \cdot p_{n ; m_{1}, m_{2}}$. Within the same time interval in the opposite direction, i.e. from point $m_{2} \cdot \Delta x$ $\left(m_{2} \in \mathbb{Z}\right)$ to point $m_{1} \cdot \Delta x\left(m_{1} \in \mathbb{Z} ; m_{1} \neq m_{2}\right)$, it is moving $U\left(m_{2}, n\right) \cdot p_{n ; m_{2}, m_{1}}$ number of vehicles.

Hence, it is possible to write down "vehicle balance" using the following recurrence relation:

$$
\begin{aligned}
& U\left(m_{1}, n+1\right) \approx U\left(m_{1}, n\right)-U\left(m_{1}, n\right) \cdot \sum_{\substack{m_{2} \in \mathbb{Z} \\
m_{2} \neq m_{1}}} p_{n ; m_{1}, m_{2}}+ \\
& \sum_{\substack{m_{2} \in \mathbb{Z} \\
m_{2} \neq m_{1}}}\left\{U\left(m_{2}, n\right) \cdot p_{n ; m_{2}, m_{1}}\right\}=\sum_{m_{2} \in \mathbb{Z}}\left\{U\left(m_{2}, n\right) \cdot p_{n ; m_{2}, m_{1}}\right\} .
\end{aligned}
$$

Then,

$$
U\left(m_{1}, n+1\right) \approx \sum_{m_{2} \in \mathbb{Z}}\left\{U\left(m_{2}, n\right) \cdot p_{n ; m_{2}, m_{1}}\right\}, \forall m_{1} \in \mathbb{Z} .
$$

The meaning of this equation is obvious: any vehicle within the studied traffic flow at the time moment $(n+1) \cdot \Delta t\left(n \in \mathbb{Z}_{+}\right) \quad$ must be coming from somewhere to the point having coordinates $m_{1} \cdot \Delta x\left(m_{1} \in \mathbb{Z}\right)$. From (3) it is obvious that the discrete model (1) is a special case of the discrete model (3). Indeed, for the $\forall m_{1}, m_{2} \in \mathbb{Z}$ in (3) assuming that

$$
p_{n ; m_{2}, m_{1}}=\left\{\begin{array}{l}
\frac{1}{2}, \text { if }\left|m_{1}-m_{2}\right|=1 ; \\
0, \text { if }\left|m_{1}-m_{2}\right| \neq 1,
\end{array}\right.
$$

the recurrent formula (1) could be received.

Similarly, to how in work [10] when receiving continuous model

$$
\frac{\partial \rho(x, t)}{\partial t}=\lim _{\substack{\Delta x \rightarrow 0 \\ \Delta t \rightarrow 0}}\left(\frac{\Delta x}{\sqrt{2 \cdot \Delta t}}\right)^{2} \cdot \frac{\partial^{2} \rho(x, t)}{\partial x^{2}}
$$

limit transitions $\Delta x \rightarrow 0, \Delta t \rightarrow 0$ in discrete model (1) have been carried out, in this section in (3) there will be also carried out limit transitions $\Delta x \rightarrow 0, \Delta t \rightarrow 0$. In this connection it is essential to distinguish between two possible scenarios:

(A) If the average "jump" of each vehicle in the studied traffic flow at a single time step also tends to zero at $\Delta x \rightarrow 0, \Delta t \rightarrow 0$, then the limit transitions
$\Delta x \rightarrow 0, \Delta t \rightarrow 0$ in the discrete model (3) will lead again to a differential equation with respect to the required density $\rho(x, t)$ of the traffic flow, and in this equation there will be "bound "only those vehicles of the flow, which form a kind of continuum, where vehicles do not interact directly, but are "infinitely close" to each other. For example, if a discrete function $p_{n ; i, j}$ is independent of $n \in \mathbb{Z}_{+}$and has the form $p_{i, j}=p(i-j)(i, j \in \mathbb{Z})$, then the average "jump" of each vehicle in the considered traffic flow for a single time step tends to zero;

(B) If the average "jump" of each vehicle at a single time step does not have to tend to zero at $\Delta x \rightarrow 0, \Delta t \rightarrow 0$, then the limit transition $\Delta x \rightarrow 0, \Delta t \rightarrow 0$ in the discrete model (3) will lead to the integral (resulting) ratio with respect to the required density $\rho(x, t)$ of the traffic flow, i.e., in this case at $\Delta x \rightarrow 0, \Delta t \rightarrow 0$, sums will turn to integrals, but not into partial derivatives.

We will not dwell on the scenario (A), and examine the scenario (B). However, after the study of scenario (B), we will return to the scenario (A) in two different ways, namely, in the results obtained for the scenario (B) we will assume at once that the scenario (A) takes place.

So, let the scenario (B) takes place. Let us rewrite the discrete model (3) as follows:

$$
\begin{aligned}
& U\left(m_{1}, n+1\right)-U\left(m_{1}, n\right) \approx \\
& \sum_{\substack{m_{2} \in \mathbb{Z} \\
m_{2} \neq m_{1}}}\left\{U\left(m_{2}, n\right) \cdot p_{n ; m_{2}, m_{1}}\right\}-U\left(m_{1}, n\right) \cdot \sum_{\substack{m_{2} \in \mathbb{Z} \\
m_{2} \neq m_{1}}} p_{n ; m_{1}, m_{2}} \cdot
\end{aligned}
$$

Having divided this equation by $\Delta t$, and then introducing designations

$$
\begin{aligned}
& x=\lim _{\substack{\Delta x \rightarrow 0 \\
m_{1} \rightarrow \pm \infty}} m_{1} \cdot \Delta x ; y=\lim _{\substack{\Delta x \rightarrow 0 \\
m_{2} \rightarrow \pm \infty}} m_{2} \cdot \Delta x ; t=\lim _{\substack{\Delta t \rightarrow 0 \\
n \rightarrow \pm \infty}} n \cdot \Delta t ; \\
& \rho(x, t)=\frac{m_{\text {weight }}}{\Delta x} \cdot U(m, n),
\end{aligned}
$$

we obtain the following integro-differential equation for the required density $\rho(x, t)$ of the traffic flow:

$$
\begin{aligned}
& \frac{\partial \rho(x, t)}{\partial t}=\int_{-l}^{+l} K(t ; y, x) \cdot \rho(y, t) d y- \\
& \rho(x, t) \cdot \int_{-l}^{+l} K(t ; x, y) d y ; x \in(-l,+l), t \in(0, T],
\end{aligned}
$$

where the kernel $K\left(t ; z_{1}, z_{2}\right) \geq 0, \quad t \in[0, T]$, $z_{i} \in[-l,+l](i=1,2) \quad$ of integro-differential equation (5) is interpreted as follows: the probability that the vehicle in the traffic flow, which at the time moment $t \in[0, T]$ was at the point $z_{1} \in[-l,+l]$ of a road section, during the next time moment $t+\Delta t$ will appear in the interval $\left[z_{2}, z_{2}+d z_{2}\right] \subset[-l,+l]$ 
$\left(z_{1} \neq z_{2}\right)$, will be equal to the value $K\left(t ; z_{1}, z_{2}\right) d z_{2} d t$. In other words, the kernel $K\left(t ; z_{1}, z_{2}\right)$ is defined as a vehicles "jump" probability density in a traffic flow from the point $z_{1} \in[-l,+l]$ to the point $z_{2} \in[-l,+l]\left(z_{1} \neq z_{2}\right)$ in a time moment $t \in[0, T]$. In other words, the function $K\left(t ; z_{1}, z_{2}\right)$ is the relative velocity of such a "jump" of vehicles in the traffic flow at time moment forms out 1D mathematical model of the nondeterministic motion "without preference" of a traffic flow, where the required function $\rho(x, t)$ is a density of a traffic flow.

\section{INVESTIGATION OF THE CONSTRUCTED MODEL (5), (6)}

Let us recall that the mathematical model (5), (6) has been obtained only under the assumption that scenario (B) takes place. Therefore, it is interesting to find out the "automatic" presence (or absence) of the scenario (A) in the mathematical model. In fact, this aspiration means that we would like to find out the catholicity of a mathematical model (5), (6) among the similar traffic flow model classes aimed at finding of the traffic flow density characteristic. It is obvious Then for $\forall y \in[-l,+l]$ we can write the identity

$$
\int_{-l}^{+l}\left\{K(t ; y, x)-\delta(x-y) \cdot \int_{-l}^{+l} K(t ; x, \xi) d \xi\right\} d x \equiv 0 .
$$

By denoting

$$
\tilde{K}(t ; y, x) \stackrel{\text { def }}{\equiv} K(t ; y, x)-\delta(x-y) \cdot \int_{-l}^{+l} K(t ; x, \xi) d \xi
$$

let us take into account identity (7) in equation (5):

$$
\frac{\partial \rho(x, t)}{\partial t}=\int_{-l}^{+l} \tilde{K}(t ; y, x) \cdot \rho(y, t) d y .
$$

It is obvious that the equations (5) and (8) are equivalent and, therefore, the model (8), (6) is also a 1D mathematical model of the traffic flow nondeterministic motion "without preference" in respect to required density and it is constructed in the assumption that the scenario (B) takes place. Now in (8) we will assume that the scenario (A) takes place. Then at any fixed $x$ and $t$ values the kernel $\tilde{K}(t ; y, x)$ of the equation (8) as function of one argument $y$ is different from zero only in the small vicinity of a point $x=x_{0} \in[-l,+l], t=t_{0} \in[0, T]$

$$
\tilde{K}\left(t_{0} ; y, x_{0}\right)=\left\{\begin{array}{l}
\bar{K}(y) \neq 0, y \in B\left(\varepsilon ; x_{0}\right), \\
0, y \notin B\left(\varepsilon ; x_{0}\right),
\end{array}\right.
$$

where $B\left(\varepsilon ; x_{0}\right) \stackrel{\text { def }}{\equiv}\left\{x: \forall \varepsilon>0\left|x-x_{0}\right|<\varepsilon\right\}$ denotes the small vicinity of a point $x_{0}$. Hence, the scenario (A) suggests that in (8) the main contribution to the $t$. It is important to note that in (5) (hereinafter and throughout this paper), the value $T$ may be equal to infinity, and consequently, in this case, instead of the interval $[0, T]$ and the half interval $(0, T]$ it is necessary to take $(0, \infty]$ and $(0, \infty)$, respectively.

Thus, integro-differential equation (5), together with the initial condition

$$
\left.\rho(x, t)\right|_{t=0}=\rho_{0}(x), x \in[-l,+l]
$$

that if the model (5), (6) will appear to be more general, than, for example, the continuous model (4) describing the traffic motion "with equiprobable preference", then there comes out a question of finding sufficient conditions under which transition from the model (5), (6) to other models, in particular, to model (4) becomes possible. In this section these and other questions are studied.

In a well-known formula $f(y)=\int \delta(x-y) \cdot f(x) d x$, where $\delta(\cdot)$ is a delta Dirac's function, let us select as function $f(\bullet)$ the following function $\quad f(\cdot) \equiv \int_{-l}^{+l} K(t ; \cdot, \xi) d \xi$. integral is carried out in $B(\varepsilon ; x)$ by the variable $y$. Let us expand the density function $\rho(y, t)$ in a Taylor series at the point $x$ :

$$
\rho(y, t)=\sum_{i=0}^{\infty} \frac{\partial \rho^{i}(x, t)}{\partial x^{i}} \cdot \frac{(y-x)^{i}}{i !} .
$$

Substituting (10) into the right-hand side of the integro-differential equation (8) gives us the following integro-differential equation:

$$
\begin{aligned}
\frac{\partial \rho(x, t)}{\partial t}=\sum_{i=1}^{\infty} & \frac{1}{(2 \cdot i) !} \cdot \frac{\partial \rho^{2 \cdot i}(x, t)}{\partial x^{2 \cdot i}} \times \\
& \int_{-l}^{+l} K(t ;|y-x|) \cdot(y-x)^{2 \cdot i} d y,
\end{aligned}
$$

where the record $K(t ;|y-x|)=K(t ; y, x)$ is legitimate by virtue of the fourth assumption from the introduction section (assumption of independence of density of the uniform traffic flow on the direction of vehicles motion). From the obtained equation (11), it is now easy to derive the 1D mathematical model (4). Indeed, from (11) follows:

$$
\begin{aligned}
& \frac{\partial \rho(x, t)}{\partial t}=\frac{1}{2} \cdot \frac{\partial \rho^{2}(x, t)}{\partial x^{2}} \cdot \int_{-l}^{+l} K(t ;|y-x|) \cdot(y-x)^{2} d y+ \\
& \sum_{i=2}^{\infty} \frac{1}{(2 \cdot i) !} \cdot \frac{\partial \rho^{2 \cdot i}(x, t)}{\partial x^{2 \cdot i}} \cdot \int_{-l}^{+l} K(t ;|y-x|) \cdot(y-x)^{2 \cdot i} d y= \\
& \frac{1}{2} \cdot \frac{\partial \rho^{2}(x, t)}{\partial x^{2}} \cdot \int_{-l}^{+l} K(t ;|z|) \cdot z^{2} d z+o\left((y-x)^{3}\right)=
\end{aligned}
$$


$a^{2}(t) \cdot \frac{\partial \rho^{2}(x, t)}{\partial x^{2}}+o\left((y-x)^{3}\right)$,

where

$$
a^{2}(t) \stackrel{\operatorname{def}}{\equiv} \int_{0}^{+l} K(t ; z) \cdot z^{2} d z>0 .
$$

It is obvious that having the assumption (9), we can neglect the terms of higher order of smallness $o\left((y-x)^{3}\right)$, and then we receive the Fokker-Planck equation (for instance, see $\frac{\partial \rho(x, t)}{\partial t}=a^{2}(t) \cdot \frac{\partial^{2} \rho(x, t)}{\partial x^{2}}$. It is obvious that the discrete model (4) is a special case of the FokkerPlanck equation: $a^{2}=\lim _{\substack{\Delta x \rightarrow 0 \\ \Delta t \rightarrow 0}}\left(\frac{\Delta x}{\sqrt{2 \cdot \Delta t}}\right)^{2}=\frac{h^{2}}{2 \cdot \tau}>0$.

It is important to highlight that the method by which model (4) was obtained from equation (11) allows other continuous 1D models to be obtained from the same equation (11) (for instance, see [2], [3], [11], [12] and appropriate references given in these). In this sense the equation (11) is the general equation (naturally, within similar/comparable classes of models) for determination of required density of a traffic flow. It should be noted that the designation (12) is a purely formal designation, since we have not clarified the question of the convergence of the integral on the right-hand side of this notation. Let us study this question. In order to do this, let us first note that equation (5) (or (8)) implies straight only convergence of the integral $\int_{0}^{+l} K(t ; z) d z$, and based on that we do not have the right to assert the convergence of the integral $\int_{0}^{+l} K(t ; z) \cdot z^{2} d z$. Therefore, there arises a question of the decrease rate of a kernel $K(t ; z)$ with a growth of $z$ for each fixed parameter $t=t_{0} \in(0, T]$. In other words, it is necessary to find a condition under which influence of a kernel $K\left(t=t_{0} ; z\right)$ would be concentrated in the small vicinity of zero. To find this required condition, we note that on the right-hand side of equation (11) for each value $i=2,3, \ldots$ ignoring the term

$$
\frac{1}{(2 \cdot i) !} \cdot \frac{\partial \rho^{2 \cdot i}(x, t)}{\partial x^{2 \cdot i}} \cdot \int_{-l}^{+l} K(t ;|y-x|) \cdot(y-x)^{2 \cdot i} d y
$$

is possible only in case if

$$
\begin{aligned}
& 2 \cdot i \cdot\left|\frac{\partial \rho^{2 \cdot(i-1)}(x, t)}{\partial x^{2 \cdot(i-1)}}\right| \int_{-l}^{+l} K(t ;|y-x|)(y-x)^{2 \cdot(i-1)} d y \gg \\
& \frac{1}{2 \cdot i-1} \cdot\left|\frac{\partial \rho^{2 \cdot i}(x, t)}{\partial x^{2 \cdot i}}\right| \int_{-l}^{+l} K(t ;|y-x|)(y-x)^{2 \cdot i} d y .
\end{aligned}
$$

Hence, for the legitimacy of the designation (12) (in other words, for the legitimacy of equation (4)) it is sufficient that for $\forall x \in[-l,+l]$ and $\forall t \in(0, T]$ the relation would be fulfilled

$$
\begin{aligned}
&\left|\frac{\partial \rho^{2}(x, t)}{\partial x^{2}}\right| \cdot \int_{0}^{+l} K(t ; z) z^{2} d z \gg \\
& \frac{1}{12} \cdot\left|\frac{\partial \rho^{4}(x, t)}{\partial x^{4}}\right| \cdot \int_{0}^{+l} K(t ; z) z^{4} d z .
\end{aligned}
$$

Since in the resulting relation (13) there appears a functions $\left|\frac{\partial \rho^{i}(x, t)}{\partial x^{i}}\right|(i=2,4)$, this relation is of little use from the practical point of view: in explicit problems it is not possible to verify the fulfilment of condition (13) because of the unknown function $\rho(x, t)$. However, in many cases it is easy to measure the averaged range of density variation of a homogeneous traffic flow and, using this characteristic of the studied transport system, from relation (13) it is easy to establish from a practical point of view a sufficient condition for a transition from a complex model (11) to a relatively simple model (4). Indeed, taking the value $\Delta x$ as the averaged interval of the vehicle "jump" in the traffic flow and taking $\Delta \rho$ as the averaged density changes range of the homogeneous traffic flow on given "jump" interval $\Delta x$ we can assume that the functions $\left|\frac{\partial \rho^{i}(x, t)}{\partial x^{i}}\right|(i=2,4)$ have orders $\frac{\Delta \rho}{(\Delta x)^{i}}$. Therefore, instead of relation (13), we can write down a new relation

$$
12 \cdot(\Delta x)^{2} \cdot \int_{0}^{+l} K(t ; z) z^{2} d z \gg \int_{0}^{+l} K(t ; z) z^{4} d z
$$

Despite the fact that the relations (13) and (14) seem to be similar, however, there are essential distinctions between them: first, the relation (13), unlike the relation (14), does not contain unknown functions, and, consequently, from the practical point of view, the condition (14) is much more preferable, since it could be easily verified; secondly, the relation (14) binds the kernel $K(t ; y, x)$ in the non-local way to the averaged range of variation in the density of a homogeneous traffic flow, and from the relation (13) this could not be concluded, since functions $\left|\frac{\partial \rho^{i}(x, t)}{\partial x^{i}}\right|(i=2,4)$, being present in that are unknown, and hence, are not being subject to comparative analysis.

Finally, it should be noted that the found sufficient condition (14) also makes it possible to discover the following interesting property of 
equation (11): even if the kernel $K(t ;|y-x|)$ of the integro-differential equation (11) remains unchanged (see the probabilistic interpretation of the kernel, which has been made immediately after the derivation of the equation 5)) for two traffic flow problems, in one of which the value $\Delta x$ (that is, the average interval of the vehicle "jump" in the traffic flow) is comparatively less than the corresponding value $\Delta x$ in another problem, then the integrodifferential equation (11) can lead to completely different models. As it has been already mentioned in the introduction, the main physical characteristics of the traffic flow are its density $\rho(x, t)$, traffic flux $q(x, t)$ and velocity $\vartheta(x, t)$. The obvious equality $q(x, t)=\vartheta(x, t) \cdot \rho(x, t)$ shows that, firstly, the flux of the traffic flow is in direct proportion to both traffic density and velocity: increase / decrease the traffic flux could be reached due to both increase / decrease in density, and due to increase / decrease the velocity of vehicles within the traffic flow; secondly, knowledge of traffic density and flux is sufficient to uniquely determine the velocity of the traffic flow. Therefore, at four assumptions listed in introduction the developed mathematical model (5), (6), (14) in respect to required density of a traffic flow doesn't allow to find other two characteristics of a traffic flow: at the same assumptions, it is necessary to develop mathematical model in respect to required traffic flux or in respect to required velocity of a traffic flow. In regards to the aforesaid, in conclusion of this section it is worth pointing out that at given four assumptions listed in introduction and assuming that scenario (B) takes place, authors of this work have succeeded to construct continuous model in respect to required traffic flux $q(x, t):$ as a result, it has been obtained again an integro-differential equation in respect to required traffic flux $q(x, t)$, but having more complex structure rather than the integro-differential equation (5). Unfortunately, the imposed restriction on the length of this article does not allow us to outline the course and result of the development process for this mathematical model.

\section{CONCLUSION}

In this paper, non-deterministic motion of urban traffic flow is studied under the assumption that vehicles can move both forward and backward within a traffic flow, and at each fixed time interval vehicles in the flow can change any of the current locations to any other vacant space with predefined probabilities. Based on these assumptions, a discrete model is developed, and then, using the principles of continuum mechanics, a transition is made from the developed discrete model to a continuous model with respect to the required traffic flow density. The received continuous model has the form of the
Cauchy initial-value problem for the integrodifferential equation, from which, in particular, there follows the Fokker-Planck equation. Further, in this paper, a sufficient condition is found, which first, ensures the mathematical legitimacy of the developed continuous model, and, secondly, proves the catholicity of the developed mathematical model among the similar classes of traffic flow models used for determining the density of the traffic flow: it is proved that fulfilment of the found condition is sufficient to obtain (under the same assumptions) other models for finding the density of the traffic flow based on the developed continuous model.

\section{ACKNOWLEDGMENTS}

For the first author the present article was partly supported by the Ventspils University College grant. For the second co-author the present article was executed within the framework of The State Research Programme "Next generation Information and Communication Technologies" ("NextIT"), Project No. 4.

\section{REFERENCES}

[1] F. A. Haight, Mathematical Theories of Traffic Flow. New York, USA: Academic Press, 1963, xi+242 pp.

[2] D. Helbing, "Traffic and related self-driven many-particle systems", Reviews of Modern Physics, vol. 73, pp. 10671141, 2001.

[3] R. Mahnke, J. Kaupuzs, and I. Lubashevsky, "Probabilistic description of traffic flow", Physics Reports, vol. 408, pp. 1130, 2005.

[4] N. H. Gartner, "Traffic Flow Theory", Transportation Research Board, Special Report 165, World Scientific Press, $1992,365 \mathrm{pp}$.

[5] C. F. Daganzo, Fundamentals of Transportation and Traffic Operations. New York, USA: Pergamon Press, 1997, 356 pp.

[6] B. S. Kerner, "Three-Phase Traffic Theory and Highway Capacity", Physica A, vol. 333, pp. 379-440, 2004.

[7] K. Nagel, P. Wagner, and R. Woesler, "Still flowing: Approaches to traffic flow and traffic jam modeling", Journal of Operations Research, vol. 51, No. 5, pp. 681-710, 2003.

[8] C. F. Daganzo, "A Behavioral Theory of Multi-Lane Traffic Flow. Part I: Long Homogeneous Freeway Sections", Transportation Research, Part B: Methodological, vol. 36, No. 2, pp. 131-158, 2002

[9] C. F. Daganzo, "A Behavioral Theory of Multi-Lane Traffic Flow Part II: Merges and the Onset of Congestion", Transportation Research, Part B: Methodological, vol. 36, No. 2, pp. 159-169, 2002.

[10] A. V. Berezhnoy, "Investigation of the traffic flow models managing parameters influence on the efficiency of the urban traffic control", Doctoral Thesis, Transport and Telecommunication Institute, Riga, Latvia, 2008, 256 pp.

[11] N. N. Smirnov, A. B. Kiselev, V. F. Nikitin, and M. V. Yumashev, Mathematical Theory of Traffic Flow. Moscow, Russian Federation: Lomonosov Moscow State University Press, 1999, $30 \mathrm{p}$.

[12] V. I. Shvetsov and D. Helbing, "Macroscopic dynamics of multilane traffic", Physical Reviews E., vol. 59, 1999, pp. 6328-6339.

[13] H. Risken and T. Frank, The Fokker-Planck Equation: Methods of Solution and Applications. Berlin, Germany: Springer-Verlag, 1989, xiv+472 p. 